\title{
Cost of organ/space infection in elective colorectal surgery. Is it just a problem of rates?
}

\author{
E Shaw ${ }^{1 *}$, A Gomila ${ }^{1}$, M Piriz ${ }^{2}$, F Obradors ${ }^{3}$, R Escofet ${ }^{1}$, R Vazquez $^{4}$, JM Badia ${ }^{4}$, L Martin $^{5}$, D Fraccalvieri ${ }^{1}$, \\ M Brugués ${ }^{6}, M C$ Nicolás ${ }^{7}$, E Espejo ${ }^{8}$, A Castro $^{9}$, A Cruz ${ }^{10}$, E Limón $^{11}$, F Gudiol $^{11}$, M Pujol ${ }^{1}$, \\ VINCat Program.Catalonia. Spain ${ }^{1}$
}

From 3rd International Conference on Prevention and Infection Control (ICPIC 2015) Geneva, Switzerland. 16-19 June 2015

\section{Introduction}

Organ/space $(\mathrm{O} / \mathrm{S})$ infection in colorectal surgery remains a major health problem. In Catalonia, the VINCat Program has monitored 24,832 procedures during 2007-2014, showing a steady rate of O/S infection over the years, 8.2\% (95\% CI 7.9 - 8.6). Improving awareness of stakeholders could be an easy strategy for assembling quality programs within health systems.

\section{Objectives}

Evaluating excess costs of organ/space infections associated with elective colorectal surgery in the Catalan Health System.

\section{Methods}

We selected a sample of 10 different sized hospitals that provided data to VINCat from January 2012-June 2014. To estimate the excess of cost we based on differences between lengths of stay in patients with and without $\mathrm{O} /$ $\mathrm{S}$ infection and extra-cost related to readmission/need of ICU and re-operation.

\section{Results}

A total of 2276 patients underwent elective colorectal surgery. O/S infection occurred in 193 (8.5\%). Patients with $\mathrm{O} / \mathrm{S}$ were more frequently men $(73 \%$ versus $60 \% ; \mathrm{p}=$ $0.001)$; underwent a rectal procedure $(43 \%$ versus $31 \%$; $\mathrm{p}<0.001)$ and had NNIS index $\geq 1(43 \%$ versus $34 \%$; $=$ 0.06 ). Median length of stay was 3 fold higher (22 days versus 7 days; $\mathrm{p}<0.001$ ) when $\mathrm{O} / \mathrm{S}$ occurred which accounted for an extra cost of $€ 3,052$ per patient. Within the group of $\mathrm{O} / \mathrm{S}$ infection, 45/193(23\%) patients were re-admitted with a median length of stay of 13 days [IQR 8 - 17)]; 117/193 (60\%) required re-operation and 56/193 (29\%) required intensive care unit stay with a median length of stay of 5 days [IQR 3 - 12]. This added an additional cost of $€ 2,235$ per patient. Accordingly, $193 \mathrm{O} / \mathrm{S}$ infections accounted for an overall excess cost of $€ 1,020,391$.

\section{Conclusion}

$\mathrm{O} / \mathrm{S}$ infection represents an important excess of cost for the Catalan Health System. The reinforcement of quality strategies can lead to a strong reduction in the use of resources and relieve its current financial constraints.

\section{Disclosure of interest}

None declared.

\section{Authors' details}

'Hospital Universitari de Bellvitge, L'Hospitalet de Llobregat, Barcelona, Spain. ${ }^{2}$ Hospital Parc Taulí, Sabadell, Barcelona, Spain. ${ }^{3}$ Fundació Althaia de Manresa, Manresa, Barcelona, Spain. ${ }^{4}$ Fundació Hospital Asil de Granollers, Granollers, Barcelona, Spain. ${ }^{5}$ Hospital de Viladecans, Viladecans, Barcelona, Spain. ${ }^{6}$ Consorci Sanitari de I'Anoia, Igualada, Barcelona, Spain. ${ }^{7}$ Hospital Universitari Mutua de Terrassa, Terrassa, Barcelona, Spain. ${ }^{8}$ Consorci Sanitari de Terrassa, Terrassa, Barcelona, Spain. ${ }^{9}$ Hospital Universitari Sant Joan de Reus, Reus, Tarragona, Spain. ${ }^{10}$ Hospital General-Parc Sanitari Sant Joan de Déu, Saint Boi de Llobregat, Barcelona, Spain. ${ }^{11}$ VINCat Program, Departament de Salut, Hospitalet de Llobregat, Barcelona, Spain.

Published: 16 June 2015

\section{doi:10.1186/2047-2994-4-S1-P77}

Cite this article as: Shaw et al: Cost of organ/space infection in elective colorectal surgery. Is it just a problem of rates? Antimicrobial Resistance and Infection Control 2015 4(Suppl 1):P77.

${ }^{1}$ Hospital Universitari de Bellvitge, L'Hospitalet de Llobregat, Barcelona, Spain Full list of author information is available at the end of the article 